\title{
Decreasing Delirium through Music (DDM) in critically ill, mechanically ventilated patients in the intensive care unit: study protocol for a pilot randomized controlled trial
}

Sikandar H. Khan ${ }^{1,4^{*}}$, Sophia Wang ${ }^{2,3}$, Amanda Harrawood ${ }^{4}$, Stephanie Martinez ${ }^{4}$, Annie Heiderscheit ${ }^{5}$, Linda Chlan ${ }^{6}$, Anthony J. Perkins ${ }^{3,7}$, Wanzhu Tu ${ }^{8}$, Malaz Boustani ${ }^{3,4,7,9,10}$ and Babar Khan ${ }^{1,4,9}$

\begin{abstract}
Background: Delirium is a highly prevalent and morbid syndrome in intensive care units (ICUs). Changing the stressful environment within the ICU via music may be an effective and a scalable way to reduce the burden of delirium.

Methods/design: The Decreasing Delirium through Music (DDM) study is a three-arm, single-blind, randomized controlled feasibility trial.

Sixty patients admitted to the ICU with respiratory failure requiring mechanical ventilation will be randomized to one of three arms (20 participants per arm): (1) personalized music, (2) non-personalized relaxing music, or (3) attention-control. Music preferences will be obtained from all enrolled participants or their family caregivers. Participants will receive two 1-h audio sessions a day through noise-cancelling headphones and $\mathrm{mp} 3$ players. Our primary aim is to determine the feasibility of the trial design (recruitment, adherence, participant retention, design and delivery of the music intervention). Our secondary aim is to estimate the potential effect size of patient-preferred music listening in reducing delirium, as measured by the Confusion Assessment Method for the ICU (CAM-ICU). Participants will receive twice daily assessments for level of sedation and presence of delirium. Enrolled participants will be followed in the hospital until death, discharge, or up to 28 days, and seen in the Critical Care Recovery Clinic at 90 days.
\end{abstract}

Discussion: DDM is a feasibility trial to provide personalized and non-personalized music interventions for critically ill, mechanically ventilated patients. Our trial will also estimate the preliminary efficacy of music interventions on reducing delirium incidence and severity.

Trial registration: ClinicalTrials.gov, Identifier: NCT03095443. Registered on 23 March 2017.

Keywords: Delirium, Music, Critical care

\footnotetext{
* Correspondence: sikhan@iu.edu

'Division of Pulmonary, Critical Care, Sleep and Occupational Medicine,

Department of Medicine, Indiana University School of Medicine, Indianapolis,

IN, USA

${ }^{4} \mathrm{IU}$ Center of Aging Research, Regenstrief Institute, Indianapolis, IN, USA

Full list of author information is available at the end of the article
} 


\section{Background}

Delirium is characterized by a disturbance of consciousness, the presence of inattention, disorganized thinking, and a fluctuating course [1]. It has an incidence as high as $85 \%$ in mechanically ventilated patients, and is associated with longer hospital stays, increased hospital and post-discharge mortality as well as higher healthcare costs [2-6]. Furthermore, over $30 \%$ of patients with delirium experience long-term cognitive impairment [7].

Despite the incidence and morbidity associated with delirium, effective pharmacological and non-pharmacological interventions to treat this condition are lacking. Music listening is a unique, non-pharmacological intervention that may reduce delirium incidence and severity through the inhibition of inflammation. Prior music listening research has demonstrated a calming effect with slow-tempo music. This is hypothesized to be secondary to entrainment of the autonomic nervous system, reduction in sympathetic activation and dampening of the inflammatory state [8]. With various pathophysiological models for delirium implicating neuro-inflammation, neurotransmitter imbalances and an aberrant stress reaction, [9] this makes music listening a promising intervention to prevent or treat delirium.

Music intervention studies in the intensive care unit (ICU) have focused on its effects on anxiety, pain, tolerance of non-invasive ventilation, and physiological indicators of the stress response (heart rate, blood pressure, catecholamines, cortisol), but, to date, studies on the effect of music listening on ICU-related delirium have not been conducted [10-16]. Our study aims to test the feasibility of music listening, and estimate the potential effect size of music listening on delirium, in critically ill patients on mechanical ventilation.

\section{Methods/design}

\section{Rationale}

We are conducting a feasibility trial to deliver personalized music for critically ill patients based on preferences supplied by their surrogates or family members. Participants in the study are unlikely to be able to communicate or choose their own music due to invasive mechanical ventilation, the need for high ventilator support, and hemodynamic instability. Our study design also includes single blinding and three intervention arms (personalized music, non-personalized, investigator-selected music, and attention-control) which adds complexity to the unique ICU environment.

Our study aims to gather data to help inform a definitive randomized controlled trial.

\section{Outcomes}

Our study aims to answer the following question: In critically ill patients on mechanical ventilation, is the design and implementation of music listening feasible?
The primary outcomes of our study are: (1) to assess recruitment rates, described by the number of participants approached and their rates of consent, (2) to assess adherence to the intervention by number of listening sessions completed per patient, and (3) to assess retention by the number of participants completing the study.

The secondary outcome in the study is to estimate the potential effect size of patient-preferred music in reducing delirium incidence as measured by the Confusion Assessment Method for the ICU (CAM-ICU).

\section{Design}

DDM is a feasibility study with a three-arm, single-blind, randomized controlled design.

Patients admitted to the ICU with respiratory failure requiring mechanical ventilation are eligible. Sixty participants will be randomized to one of three arms (20 patients per arm): (1) personalized music, (2) non-personalized relaxing music, or (3) attention-control (see Fig. 1). Participants will receive two 1 -h audio sessions a day through noise-cancelling headphones (and mp3 players). We chose an attention-control arm (rather than usual care) to test the feasibility of a placebo, and to control for the non-specific effects of headphone and non-audio sounds in the study.

The trial protocol was written according to the Standard Protocol Items: Recommendations for Interventional Trials Statement (SPIRIT). A SPIRIT Figure is provided (see Fig. 2) and a SPIRIT Checklist is included as Additional file 1.

\section{Setting}

Patients admitted to the medical or surgical ICU service at Sidney and Lois Eskenazi Hospital (Indianapolis, Indiana) are eligible. Eskenazi Hospital is a busy 618-bed, urban, safety net hospital with 44 ICU beds (22 surgical, 22 medical), averaging 120 ICU admissions per month. Demographics of this ICU are: mean age of patients 53.7 years, 45\% African American, 47\% female, 29\% age 60 years or older, and $16 \%$ with Medicaid insurance.

\section{Participants}

The target population for our trial is adults aged 18 years and older, admitted to the medical or surgical ICU on invasive mechanical ventilation.

\section{Consent}

Individuals deemed eligible for the study will be approached for consent. If on mechanical ventilation and unable to consent, the patient's surrogate or healthcare representative will be approached for consent.

\section{Inclusion criteria}

Inclusion criteria for our study are: (1) age 18 years or older, (2) English speaking, (3) admitted to the ICU 


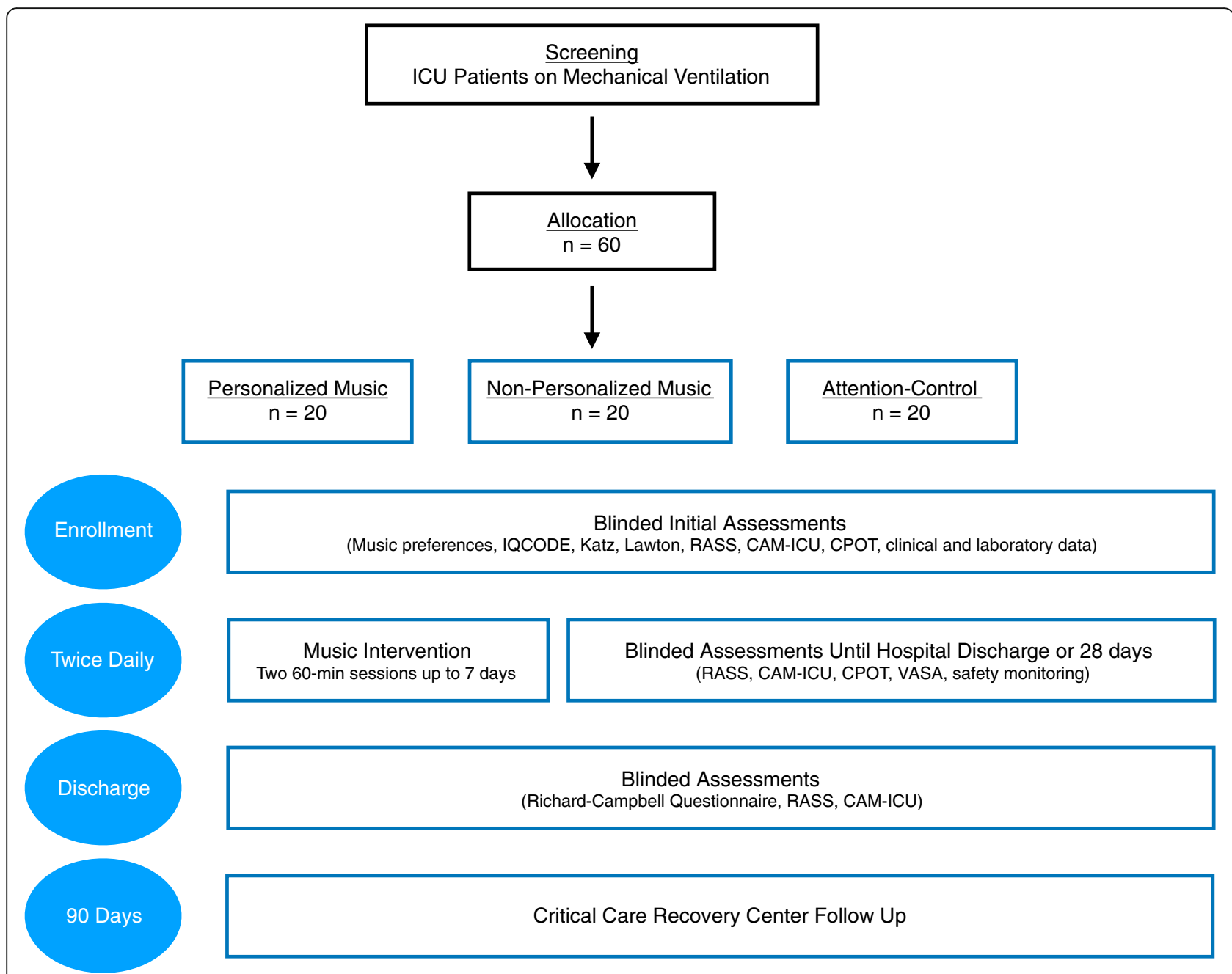

Fig. 1 DDM study flowchart, interventions, and assessments. Abbreviations: CAM-ICU Confusion Assessment Method for the Intensive Care Unit, CPOT Critical Pain Observation Tool, Katz Katz Index of Independence in Activities of Daily Living, Lawton Lawton Instrumental Activities of Daily Living, RASS Richmond Agitation-Sedation Scale, VASA Visual Analog Scale for Anxiety

(medical or surgical), and (4) receiving mechanical ventilation for an anticipated duration of $24 \mathrm{~h}$ or longer, provided that the patient has not already been on the ventilator longer than $48 \mathrm{~h}$ at the time of enrollment.

\section{Exclusion criteria}

Patients are excluded from our study if any of the following conditions are present: (1) severe dementia or chronic neurological disease, with an IQCODE score of 4.5 or greater; [17-19] (2) acute or subacute severe neurological deficit or injury; (3) severe psychiatric illness (not including depression) or developmental problems; (4) suspected or confirmed drug or alcohol intoxication/overdose or withdrawal; (4) uncorrected or severe hearing or vision impairment; (5) coma after cardiac arrest and/or hypothermia protocol; (6) pregnant or nursing women; (7) prisoners; (8) expected death within $24 \mathrm{~h}$ of enrollment or lack of commitment to aggressive treatment by family or the medical team; (9) inability to obtain consent from legally authorized representative within $72 \mathrm{~h}$ of meeting eligibility; and (10) attending physician or healthcare team refusal.

\section{Materials}

Participants are provided with noise-cancelling headphones (Maxell NC-IV Superior Noise Cancellation), with mp3 player devices (Apple iPod Shuffle 2GB, 6th generation or Goldenseller 16GB Mp3 Player) during the trial. Music tracks and audiobooks were purchased from Apple Music Store (iTunes).

\section{Study procedures}

\section{Screening}

Patients admitted to the ICU are screened on a daily basis. Music preference data will be collected from all enrolled 


\begin{tabular}{|c|c|c|c|c|c|c|c|}
\hline \multirow[b]{3}{*}{ TIMEPOINT** } & \multicolumn{7}{|c|}{ STUDY PERIOD } \\
\hline & \multirow{2}{*}{$\begin{array}{c}\text { Enrolment } \\
-t_{1}\end{array}$} & \multirow{2}{*}{$\begin{array}{c}\text { Allocation } \\
0\end{array}$} & \multicolumn{4}{|c|}{ Post-allocation } & \multirow{2}{*}{$\begin{array}{c}\text { Close-out } \\
\text { Day } 90\end{array}$} \\
\hline & & & $\begin{array}{r}\text { Day } \\
1-7 \\
\end{array}$ & $\begin{array}{l}\text { Day } \\
8-14 \\
\end{array}$ & $\begin{array}{c}\text { Day } \\
15-21 \\
\end{array}$ & $\begin{array}{c}\text { Day } \\
21-28 \\
\end{array}$ & \\
\hline \multicolumn{8}{|l|}{ ENROLMENT: } \\
\hline Eligibility screen & $\mathrm{x}$ & & & & & & \\
\hline \multirow{3}{*}{$\begin{array}{r}\text { Informed consent } \\
\text { Music preferences, } \\
\text { demographics, } \\
\text { comorbidities, } \\
\text { functional status, } \\
\text { IQCODE, } \\
\text { Allocation }\end{array}$} & $\mathrm{x}$ & & & & & & \\
\hline & $x$ & & & & & & \\
\hline & & $x$ & & & & & \\
\hline \multicolumn{8}{|l|}{ INTERVENTIONS: } \\
\hline $\begin{array}{r}\text { Personalized } \\
\text { Music }\end{array}$ & & & $2 x$ & & & & \\
\hline $\begin{array}{r}\text { Non-personalized } \\
\text { Music }\end{array}$ & & & $2 x$ & & & & \\
\hline $\begin{array}{l}\text { Attention- } \\
\text { control/audiobook }\end{array}$ & & & $2 X$ & & & & \\
\hline \multicolumn{8}{|l|}{ ASSESSMENTS: } \\
\hline $\begin{array}{r}\text { RASS, CAM-ICU, } \\
\text { CPOT }\end{array}$ & $\mathrm{x}$ & $\mathrm{x}$ & $2 x$ & $2 x$ & $2 x$ & $2 x$ & \\
\hline \multirow{2}{*}{$\begin{array}{r}\text { Faces Anxiety } \\
\text { Scale, ICU Mobility } \\
\text { Clinical data } \\
\text { (vitals, labs, vent } \\
\text { settings, imaging) }\end{array}$} & & & $\mathrm{x}$ & $x$ & $\mathrm{x}$ & $\mathrm{x}$ & $\mathrm{x}$ \\
\hline & $\mathrm{x}$ & $\mathrm{x}$ & $\mathrm{x}$ & $x$ & $\mathrm{x}$ & $\mathrm{x}$ & $x$ \\
\hline $\begin{array}{r}\text { Psychotropic } \\
\text { Medications }\end{array}$ & $x$ & $\mathrm{x}$ & $\mathrm{x}$ & $x$ & $x$ & $\mathrm{x}$ & \\
\hline $\begin{array}{r}\text { Disposition at } \\
\text { Discharge }\end{array}$ & & & $x$ & $\mathrm{x}$ & $\mathrm{x}$ & $\mathrm{x}$ & $\mathrm{x}$ \\
\hline $\begin{array}{r}\text { Readmission and } \\
\text { Death }\end{array}$ & & & & & & & $\mathrm{x}$ \\
\hline
\end{tabular}

Fig. 2 Standard Protocol Items: Recommendations for Interventional Trials (SPIRIT) Figure for the DDM Trial. Abbreviations: CAM-ICU Confusion Assessment Method for the Intensive Care Unit, CPOT Critical Pain Observation Tool, ICU intensive care unit, IQCODE Informant Questionnaire on Cognitive Decline in the Elderly, RASS Richmond Agitation-Sedation Scale

participants (through their surrogates) regardless of intervention arm.

\section{Participant randomization}

Using a computer-generated list of random numbers created by study statistician, participants are randomized by permuted block with varying block sizes. Randomization occurs within $48 \mathrm{~h}$ of enrollment in a 1:1:1 ratio, and only the unblinded study coordinator, who also programs the music devices, is aware of the randomization assignment.

\section{Study duration/termination}

The music intervention period is 7 days post randomization. Participants will stop receiving music sessions early if they choose to withdraw, are transferred out of the ICU, die, or are discharged. Inhospital follow-up continues to 28 days or until discharge, whichever occurs earlier, by daily assessment, unless they choose to withdraw. All participants will be invited for follow-up in the Critical Care Recovery Clinic at 90 days.

\section{Pre-intervention phase}

Upon enrollment, demographics, clinical data (vital signs, pertinent laboratory and imaging data), severity of illness (APACHE II and SOFA), comorbidity burden (Charlson Comorbidity Index), pertinent home medications, reason for admission, pre-hospital functional status (Katz and Lawton), and cognitive function (Informant Questionnaire on Cognitive Decline in the Elderly (IQCODE)) are obtained [20-23]. Cumulative doses of antipsychotic, analgesic, sedative, and anxiolytic medications are also recorded.

A previously described music-preference assessment tool is utilized to obtain the participants' music preferences from family members/surrogates [24]. We collect information about genre likes and dislikes, as well as specific favorite artists, tracks, and instruments. 
A pre-intervention level of consciousness and delirium assessment is performed upon enrollment through the Richmond Agitation Sedation Scale and the CAM-ICU, respectively $[3,25]$.

\section{Playlist development}

Following enrollment consent, blinded research personnel enter the participant's music preferences into a Research Electronic Data Capture (REDCap) database. The unblinded study coordinator programs music devices based on an individual study participant's randomization status. For those randomized to personalized music, Apple Music is utilized to create a playlist based on their musical interests.

Participants randomized to non-personalized music received an mp3 player programmed with slow-tempo, relaxing music and instrumental sounds (piano, relaxing rainfall sounds, and classical music). Finally, participants randomized to attention-control receive a device with audiobooks. The audiobooks include Treasure Island, Harry Potter, and Dr. Seuss' Oh the Places You'll Go!', and were chosen for their readability and for their broad appeal.

The playlist for each participant is carefully tracked, which will permit subsequent analysis should particular books or tracks be associated with increased delirium incidence or severity.

To avoid unblinding study personnel to the intervention arm, the coordinator waits a minimum of $4 \mathrm{~h}$ to release the mp3 device for use in the study. Prior to allocating devices to patient rooms, all devices are checked to ensure that identifying data including name, artist, collection, and genre have been removed, the device is clean and in good functioning order, and volume is set to a pre-specified level.

\section{Intervention phase}

Music listening sessions are twice a day, for $1 \mathrm{~h}$ at a time, from 10:00 to 11:00 and 14:00 to 15:00, for 7 days. The times are based on feedback from ICU nurses associated with least interference with clinical activities. Music listening is provided for 7 days unless the participant refuses, withdraws, is transferred out of the ICU, or dies. Participants extubated within the 7 -day period continue to receive the intervention unless they are transferred out of the ICU. Participants remaining in the ICU after 7 days will not receive further intervention due to the resource limitations of our feasibility study.

During listening sessions, a conducive listening environment is created; medical teams are asked to avoid non-urgent patient care activities, the in-room television is turned off, family members are asked to limit their interactions with the patient, and the door is closed to limit outside noise. As all three arms of the trial will receive a conducive listening environment, and due to the use of noise-cancelling headphones in these settings, we believe that the audio dose will remain as the pure active intervention.

Participants receive their first dose of music listening, in one of these windows, as soon after enrollment as possible.

The ICU clinical nurses receive on-site training in the study protocol, and in the operation of the music devices and headphones. Blinded research personnel and/or hospital nurses start the music sessions by placing the headphones on the participants and activating the audio. Adherence is monitored by recording the start and stop date and times for each session. The participant's heart rate and blood pressure pre and post session are also recorded.

All participants in the trial receive the ABCDEF bundle that is standard of care in the ICU where the study is being conducted. The ABCDEF bundled delirium care protocol places a multidisciplinary focus on daily ventilator liberation trials, sedation vacation, choice and optimization of sedation, nursing-led delirium screenings, early mobility, and family/social support [26]. Participants in the study will continue to have physician-determined sedation without input on choice of sedation from our trial.

\section{Blinding during intervention}

We are using various measures to ensure concealment of a participant's allocation. Only the unblinded study coordinator programs the audio devices, and ensures that the tracks are anonymized (renamed to a series of digits or letters), to prevent unblinding when bedside staff view the audio device's screen. Our protocol also benefits from the fact that the majority of audio devices lack screens entirely (Apple iPod Shuffle). Participants, their family members/surrogates, and hospital nurses are asked not to share a patient's randomization with anyone in the study, should they become aware of it. Audio is not turned on until the headphones are securely placed on the participant to prevent study personnel from inadvertently hearing the output. Participants are permitted to change tracks or adjust the volume if needed. Should one of the study personnel become unblinded, they are reassigned to other study participants. To assess the fidelity of these efforts, we will survey research personnel to predict patient randomization in a subset of participants.

\section{Assessments during intervention}

All assessments are performed by blinded research assistants. Assessments occur twice a day, approximately at 11:00 and 15:00 (after audio interventions). Study personnel perform the following: (1) Richmond Agitation-Sedation Scale (RASS) to document level of consciousness; (2) Confusion Assessment Method for the ICU (CAM-ICU) to screen for delirium [3, 25, 27], and CAM-ICU-7 to determine delirium severity [28]; (3) Critical Care Pain 
Observation Tool (CPOT) to determine level of analgesia and symptom control [29]; (4) Faces Anxiety Scale (performed after the morning listening session) [30, 31]; (5) physical therapy and occupational therapy notes are evaluated and each participant's mobility milestones are recorded using Hodgson Mobility Scale [32]; and (6) adverse events are assessed (including seizures while listening, localized skin breakdown or cellulitis around the ears) and are reported to the Institutional Review Board (IRB) per usual protocol.

\section{Post intervention}

Participants receive in-hospital assessments by blinded research personnel until hospital discharge, day 28 , or they withdraw or die. Twice a day, at approximately 11:00 and 15:00, study personnel perform the RASS, CAM-ICU, CAM-ICU-7, CPOT, and the Faces Anxiety Scale, evaluate mobility with the Hogdson Mobility Scale, and record adverse events.

Beginning within $72 \mathrm{~h}$ of extubation or removal of the endotracheal tube, and if the patient is CAM-ICU negative, the participant is reconsented for the study. The participant is also screened for obstructive sleep apnea with STOP-BANG and the Richards-Campbell Sleep Questionnaires, given the higher incidence of delirium in patients with sleep apnea and disturbed sleep quality [33-35].

\section{Follow-up}

Participants are invited to follow-up in the Critical Care Recovery Center (CCRC) at 90 days from hospital discharge. The CCRC is an outpatient interdisciplinary clinic comprising care coordinators (social worker, nurse), a critical care physician, psychometrician, and psychologist. Its goal is to maximize the cognitive, physical, and psychological recovery of ICU survivors through comprehensive assessments and personalized recovery plans incorporating self-management protocols.

\section{Data collection}

Prior to the trial initiation, study personnel underwent training sessions on data collection and were individually tested on data entry as well as outcome assessments.

Study data have been collected and managed using REDCap electronic data capture tools hosted at Indiana University. REDCap is a secure, web-based application designed to support data capture for research studies, providing: (1) an intuitive interface for validated data entry; (2) audit trails for tracking data manipulation and export procedures; (3) automated export procedures for seamless data downloads to common statistical packages; and (4) procedures for importing data from external sources.
On a daily basis during all phases of the study and for up to 28 days, blinded personnel collect vital signs, pertinent laboratory and imaging data, data to calculate severity of illness, clinical events including surgery and tracheostomy, duration and modes of mechanical ventilation, cumulative daily doses of sedatives, analgesics, antipsychotics, presence of shock requiring vasopressors, and the administration of systemic steroids or paralytic agents. We also collect disposition at discharge.

\section{Sample size and feasibility}

We determined criteria for feasible recruitment rates based on prior research. A large trial of music in the ICU had a recruitment rate of $33 \%$, while delirium studies have had recruitment rates close to 30\% [11]. Our own recruitment experience at the institution where this trial is being conducted suggests recruitment rates near $60 \%$. Given a sample size of 60 participants, we will be able estimate a recruitment rate of $60 \%$ to within a $95 \%$ confidence interval of $\pm 12.40 \%$. A recruitment rate of $48 \%$ would be lower than our institutional norm, but remains higher than other similar trials.

Adherence to the intervention will be defined as the percentage of audio sessions completed per patient (a maximum of 14 sessions per patient at a rate of two sessions per day for 7 days), adjusted for number of eligible sessions based on length of stay in the ICU. Our sample size of 60 participants will permit an estimate of the adherence rate of $80 \%$ to within a $95 \%$ confidence interval of $\pm 10.12 \%$. We determined a retention rate of $80 \%$ for feasibility, defined by the percentage of participants completing all in-hospital components of the study, which can be estimated with a similar confidence interval. As a pilot study, we anticipate that a sample size of 60 participants will be sufficient to determine feasibility.

Our pilot study will help calculate sample size needed for a definitive randomized controlled trial. A decrease of $15 \%$ in delirium incidence between groups would make a larger trial feasible, with a three-arm trial requiring 548 participants (allowing $20 \%$ for attrition, $\alpha=0.05$, power $=80 \%$ ).

\section{Statistical analysis}

We will use chi-square tests to determine if study retention rates differ across the three groups. Adherence will be defined as the percentage of audio sessions completed per patient, adjusted for number of eligible sessions based on length of stay in the ICU. Depending on the distribution of this outcome, we will use either analysis of variance (ANOVA) (for normally distributed data) or the Kruskal-Wallis test (non-normal data) to determine if adherence differs between the three study arms. 


\section{Discussion}

DDM is a feasibility trial for providing personalized and non-personalized music interventions for critically ill, mechanically ventilated patients. In our trial we also estimate the preliminary efficacy of music interventions on reducing delirium incidence and severity.

Our protocol builds on prior research as the anxiolytic properties of music have been well documented [12]. Music interventions for hospitalized and non-hospitalized patients have shown decreases in heart rate and blood pressure, and were hypothesized to be a consequence of a lower sympathetic drive [12, 14]. Earlier studies have also shown lower sedative doses for patients undergoing procedures and utilizing preferred music listening [11]. Prior studies have been limited by methodological challenges, including small sample size, lack of blinding, and exclusion of the critically ill. The strengths of our protocol include blinding of outcome assessors, twice daily delirium assessments, as well as assessments of pain, anxiety, and mobility milestones. Furthermore, our study benefits from high external validity through inclusion of the ICU's sickest patients.

Music listening has been shown to activate areas of the brain involved with memory, cognitive function, and emotion [36, 37]. By reducing brain dysfunction and increasing activity in the areas related to memory, music could help retain cognitive function, particularly in older people who experience critical illness or injury. Extended applications of our study could permit comparison of neurocognitive outcomes between music and attentioncontrol arms in future studies.

As a feasibility study, our protocol will enable us to perform hypothesis testing, develop music algorithms, and implement a music intervention protocol in a busy ICU. The results of this study will inform the protocol design of a larger study.

\section{Trial status}

Enrollment is ongoing. Recruitment began in December 2016 and is expected to conclude in January 2018. Target enrollment for the study is 60 participants. The trial was retroactively registered at ClinicalTrials.gov.

\section{Additional file}

Additional file 1: SPIRIT 2013 Checklist: recommended items to address in a clinical trial protocol and related documents. (DOC $122 \mathrm{~kb}$ )

\footnotetext{
Abbreviations

CAM-ICU: Confusion Assessment Method for the Intensive Care Unit; DDM: Decreasing Delirium through Music; IQCODE: Information Questionnaire on Cognitive Decline in the Elderly
}

\section{Acknowledgements}

We would like to thank Emily Behrens, the Regenstrief research team and Eskenazi Hospital for their efforts in development of this protocol.

\section{Funding}

The DDM Trial is supported by Regenstrief Institute's Innovations Grant. Regenstrief Institute did not participate in the design of this study. SK is supported by NHLBI 5T32HL091816-07.

\section{Availability of data and materials}

Data results from this feasibility study are unavailable at the time of publication. Access to the final dataset will be retained with the study investigators.

Compensation for the trial, including harm, is not intended. Results of the trial are intended for publication in a peer-reviewed journal, by the authors, and assistance of professional writers is not expected. Results will be shared with participants of the study by e-mail, and within our hospital system. Full access to the protocol or participant-level dataset is available upon request.

\section{Authors' contributions}

Study concept and design: BK. Data acquisition: SK, SM, and AH. Data analysis: AP. Drafting of manuscript: SK. Critical revision of the manuscript: SW, MB, AH, LC, WT, and BK. Statistical analysis: AP. Obtained funding: BK. Administrative support: AH. Study supervision: BK. All authors read and approved the final manuscript.

Ethics approval and consent to participate

The DDM Trial received Indiana University's Institutional Review Board approval (IRB 1608887741A003).

For participants deemed eligible for enrollment, the patient and/or their legal representative are approached for consent.

\section{Consent for publication}

Consent forms for the trial include consent for publication of results in peerreviewed journals.

\section{Competing interests}

The authors declare that they have no competing interests.

\section{Publisher's Note}

Springer Nature remains neutral with regard to jurisdictional claims in published maps and institutional affiliations.

\section{Author details}

'Division of Pulmonary, Critical Care, Sleep and Occupational Medicine, Department of Medicine, Indiana University School of Medicine, Indianapolis, IN, USA. ²Department of Psychiatry, Indiana University School of Medicine, Indianapolis, IN, USA. ${ }^{3}$ Indiana University Center of Health Innovation and Implementation Science, Indianapolis, IN, USA. ${ }^{4}$ IU Center of Aging Research, Regenstrief Institute, Indianapolis, IN, USA. ${ }^{5}$ Music Therapy Department, Augsburg College, Minneapolis, MN, USA. ${ }^{6}$ Department of Nursing, Nursing Research Division, Mayo Clinic, Rochester, MN, USA. IIndiana Clinical and Translational Research Institute, Indiana University School of Medicine, Indianapolis, IN, USA. ${ }^{8}$ Department of Biostatistics, Indiana University School of Medicine, Indianapolis, IN, USA. ${ }^{9}$ Sandra Eskenazi Center for Brain Care Innovation, Eskenazi Hospital, Indianapolis, IN, USA. ${ }^{10}$ Division of Geriatrics and General Internal Medicine, Department of Internal Medicine, Indiana University School of Medicine, Indianapolis, IN, USA.

Received: 9 May 2017 Accepted: 14 November 2017

Published online: 29 November 2017

\section{References}

1. Miller RR, Ely EW, editors. Delirium and cognitive dysfunction in the intensive care unit. Seminars in respiratory and critical care medicine. New York: Thieme; 2006.

2. Ely E, Inouye SK, Bernard GR, et al. Delirium in mechanically ventilated patients: validity and reliability of the confusion assessment method for the intensive care unit (cam-icu). JAMA 2001;286(21):2703-10.

3. Ely EW, Margolin R, Francis J, May L, Truman B, Dittus R, et al. Evaluation of delirium in critically ill patients: validation of the Confusion Assessment Method for the Intensive Care Unit (CAM-ICU). Crit Care Med. 2001;29(7):1370-9. 
4. McNicoll L, Pisani MA, Zhang Y, Ely EW, Siegel MD, Inouye SK. Delirium in the intensive care unit: occurrence and clinical course in older patients. J Am Geriatr Soc. 2003;51(5):591-8.

5. Thomason JW, Shintani A, Peterson JF, Pun BT, Jackson JC, Ely EW. Intensive care unit delirium is an independent predictor of longer hospital stay: a prospective analysis of 261 non-ventilated patients. Crit Care. 2005:9(4):R375.

6. Ely E, Shintani A, Truman B, et al. Delirium as a predictor of mortality in mechanically ventilated patients in the intensive care unit. JAMA. 2004;291(14):1753-62.

7. Jackson JC, Hart RP, Gordon SM, Shintani A, Truman B, May L, et al. Sixmonth neuropsychological outcome of medical intensive care unit patients. Crit Care Med. 2003;31(4):1226-34.

8. Beaulieu-Boire G, Bourque S, Chagnon F, Chouinard L, Gallo-Payet N, Lesur O. Music and biological stress dampening in mechanically-ventilated patients at the intensive care unit ward - a prospective interventional randomized crossover trial. J Crit Care. 2013;28(4):442-50.

9. Khan BA, Farber MO, Campbell N, Perkins A, Prasad NK, Hui SL, et al. S100 calcium binding protein $B$ as a biomarker of delirium duration in the intensive care unit—an exploratory analysis. Int J Gen Med. 2013;6:855-61.

10. Chlan L. Effectiveness of a music therapy intervention on relaxation and anxiety for patients receiving ventilatory assistance. Heart Lung. 1998;27(3):169-76.

11. Chlan LL, Weinert CR, Heiderscheit A, Tracy MF, Skaar DJ, Guttormson JL, et al. Effects of patient-directed music intervention on anxiety and sedative exposure in critically ill patients receiving mechanical ventilatory support: a randomized clinical trial. JAMA. 2013;309(22):2335-44.

12. Han L, Li JP, Sit JW, Chung L, Jiao ZY, Ma WG. Effects of music intervention on physiological stress response and anxiety level of mechanically ventilated patients in China: a randomised controlled trial. J Clin Nurs. 2010;19(7-8):978-87.

13. Hetland $B$, Lindquist $R$, Chlan LL. The influence of music during mechanical ventilation and weaning from mechanical ventilation: a review. Heart Lung. 2015;44(5):416-25.

14. Korhan EA, Khorshid L, Uyar M. The effect of music therapy on physiological signs of anxiety in patients receiving mechanical ventilatory support. J Clin Nurs. 2011:20(7-8):1026-34.

15. Lee $\mathrm{CH}$, Lee $\mathrm{CY}$, Hsu MY, Lai $C L$, Sung $Y H$, Lin $C Y$, et al. Effects of music intervention on state anxiety and physiological indices in patients undergoing mechanical ventilation in the intensive care unit. Biol Res Nurs. 2017;19(2):137-44

16. Mofredj A, Alaya S, Tassaioust K, Bahloul H, Mrabet A. Music therapy, a review of the potential therapeutic benefits for the critically ill. J Crit Care. 2016:35:195-9.

17. Jorm AF. A short form of the Informant Questionnaire on Cognitive Decline in the Elderly (IQCODE): development and cross-validation. Psychol Med. 1994;24(1):145-53.

18. Jorm AF, Jacomb PA. The Informant Questionnaire on Cognitive Decline in the Elderly (IQCODE): socio-demographic correlates, reliability, validity and some norms. Psychol Med. 1989;19(4):1015-22.

19. Jorm AF, Scott R, Cullen JS, MacKinnon AJ. Performance of the Informant Questionnaire on Cognitive Decline in the Elderly (IQCODE) as a screening test for dementia. Psychol Med. 1991;21(3):785-90.

20. Charlson ME, Pompei P, Ales KL, MacKenzie CR. A new method of classifying prognostic comorbidity in longitudinal studies: development and validation. J Chronic Dis. 1987:40(5):373-83.

21. Katz S, Ford AB, Moskowitz RW, Jackson BA, Jaffe MW. Studies of illness in the aged: The index of ADL: a standardized measure of biological and psychosocial function. JAMA. 1963;185(12):914-9.

22. Knaus WA, Draper EA, Wagner DP, Zimmerman JE. APACHE II: a severity of disease classification system. Crit Care Med. 1985;13(10):818-29.

23. Vincent JL, Moreno R, Takala J, Willatts S, De Mendonca A, Bruining H, et al. The SOFA (Sepsis-related Organ Failure Assessment) score to describe organ dysfunction/failure. On behalf of the Working Group on Sepsis-Related Problems of the European Society of Intensive Care Medicine. Intensive Care Med. 1996;22(7):707-10.

24. Chlan L, Heiderscheit A. A tool for music preference assessment in critically ill patients receiving mechanical ventilatory support. Music Ther Perspect. 2009;27(1):42-7.

25. Sessler CN, Gosnell MS, Grap MJ, Brophy GM, O'Neal PV, Keane KA, et al. The Richmond Agitation-Sedation Scale: validity and reliability in adult intensive care unit patients. Am J Respir Crit Care Med. 2002;166(10):1338-44.
26. Marra A, Ely EW, Pandharipande PP, Patel MB. The ABCDEF Bundle in critical care. Crit Care Clin. 2017:33(2):225-43.

27. Khan BA, Guzman O, Campbell NL, Walroth T, Tricker J, Hui SL, et al. Comparison and agreement between the Richmond Agitation-Sedation Scale and the Riker Sedation-Agitation Scale in evaluating patients' eligibility for delirium assessment in the ICU. Chest. 2012;142(1):48-54.

28. Khan BA, Perkins AJ, Gao S, Hui SL, Campbell NL, Farber MO, et al. The Confusion Assessment Method for the ICU-7 Delirium Severity Scale: a novel delirium severity instrument for use in the ICU. Crit Care Med. 2017;45(5):851-7.

29. Gelinas C, Fortier M, Viens C, Fillion L, Puntillo K. Pain assessment and management in critically ill intubated patients: a retrospective study. Am J Crit Care. 2004;13(2):126-35.

30. Gustad LT, Chaboyer W, Wallis M. Performance of the faces anxiety scale in patients transferred from the ICU. Intensive Crit Care Nurs. 2005:21(6):35560. Epub 2005 Aug 15.

31. McKinley S, Stein-Parbury J, Chehelnabi A, Lovas J. Assessment of anxiety in intensive care patients by using the Faces Anxiety Scale. Am J Crit Care. 2004;13(2):146-52

32. Hodgson C, Needham D, Haines K, Bailey M, Ward A, Harrold M, et al. Feasibility and inter-rater reliability of the ICU Mobility Scale. Heart Lung. 2014:43(1):19-24.

33. Chung F, Yegneswaran B, Liao P, Chung SA, Vairavanathan S, Islam S, et al STOP questionnaire: a tool to screen patients for obstructive sleep apnea. Anesthesiology. 2008;108(5):812-21.

34. Kamdar BB, Shah PA, King LM, Kho ME, Zhou X, Colantuoni E, et al. PatientNurse Interrater Reliability and Agreement of the Richards-Campbell Sleep Questionnaire. Am J Crit Care. 2012;21(4):261-9.

35. Mirrakhimov AE, Brewbaker CL, Krystal AD, Kwatra MM. Obstructive sleep apnea and delirium: exploring possible mechanisms. Sleep Breath. 2014;18(1):19-29.

36. Stubbs T. Experiences and perceptions of music therapy in critical illness. Nurs Times. 2005;101(45):34-6

37. Zhang Y, Cai J, An L, Hui F, Ren T, Ma H, et al. Does music therapy enhance behavioral and cognitive function in elderly dementia patients? A systematic review and meta-analysis. Ageing Res Rev. 2017;35:1-11.

\section{Submit your next manuscript to BioMed Central and we will help you at every step:}

- We accept pre-submission inquiries

- Our selector tool helps you to find the most relevant journal

- We provide round the clock customer support

- Convenient online submission

- Thorough peer review

- Inclusion in PubMed and all major indexing services

- Maximum visibility for your research

Submit your manuscript at www.biomedcentral.com/submit
) Biomed Central 